\title{
Principles of construction and methods of using the intellectual system of teaching and knowledge control based on multi-level hierarchical adaptive semantic models
}

\author{
Tamara Shikhnabieva ${ }^{1,2, *}$ \\ ${ }^{1}$ Institute of Education Management of the Russian Academy of Education, 105062, 5/16, 1B, \\ Makarenko str., Moscow, Russia \\ ${ }^{2}$ Plekhanov Russian of University Economics, 117997, 36 Stremyanny per., Moscow, Russia
}

\begin{abstract}
Intellectual methods and models are widely used in those subject areas, in which preliminary decision analysis requires decision support. Intellectual systems are also widely used also in education. The article considers various technologies for the development of intellectual information systems for educational purposes, as well as the principles of construction, structure and methodology for using the developed intellectual system of teaching and knowledge control based on multi-level hierarchical adaptive semantic models.
\end{abstract}

\section{Introduction}

At the present stage of the development of the education system, it is closer to that threshold, beyond which mass use of semantic technologies and intellectual teaching systems (ITS) should be expected.

Intellectualization of information systems for educational purposes is also conditioned by the need to introduce high-tech automated information systems not only in the educational process, but also directly in the management of educational institutions in order to improve its quality.

Our article is devoted to the problems arising in the choice of the model of representation and structuring of knowledge in intellectual systems of teaching and control of knowledge and some approaches to their solution. Also, the work describes the principles of construction and the methodology for using the intellectual system developed by us, based on multilevel hierarchical adaptive semantic models.

\section{Intellectualization of information systems in education: a review of the existing development technologies}

\footnotetext{
* Corresponding author: shetoma@mail.ru
} 
The review of publications on the intellectualization of information systems in education also showed that over the last decade a number of directions for the intellectualization of information systems for educational purposes have been formed [1-4, 6, 7, 8]. These areas are associated with the use of various methods, models of artificial intellect and technology in the development of intellectual information systems.

Consider the intellectualization of information systems in education in terms of the use of certain technologies that allow them to be created. These are: agent-oriented technologies, technology of expert systems, artificial neural networks, fuzzy logic, genetic algorithms and a number of others. In intellectual educational information systems, these technologies are oriented towards the modern organization of education at all its levels.

One of the types of intellectual systems includes integrated expert systems [4]. As an instrument for building such intellectual systems, including network adaptive intellectual teaching systems (ITS), the AT-TECHNOLOGY complex, developed on the basis of the Laboratory of Intellectual Systems and Technologies at the Chair of Cybernetics of the MIFI National Research Nuclear University, is of interest. As the author of [4] points out, the effective functioning of such network adaptive IOS is supported by a system dynamic modification of the software tools "using a set of unified procedures" based on the current running version of the complex. Modification of the software includes modification of the implemented algorithms, models and methods, as well as the source code and the script, including the relationship of the most modifiable component with other software. Within the framework of teaching courses in this area of teaching, the formation of heuristic models of knowledge representation is being studied. Here special attention is paid to network models of representation of knowledge, including modeling of the simplest situations of the problem area with the help of frame models of the description of knowledge and semantic networks. Currently, as a part of the tools to support the construction of the teaching model in the network version of the AT-TECHNOLOGY complex, there are a number of components for identifying learner skills to simulate the simplest situations of the domain with the help of frames and semantic networks.

Depending on the type of student model and its individual approaches to teaching (in general, approaches can be inductive, deductive and hybrid) it is suggested to use three teaching vectors (fast, normal and slow) $[1,2,4,17]$.

On this basis, the process of real teaching is simulated, taking into account such characteristic features as the mutual integration of the processes of verification of the learner's models, the teacher and the teaching course, the pupil's ability, the optimal strategy for dosing knowledge and exercises by the teacher, the speed of memorizing and forgetting the student's knowledge, the duration and stability of his active condition and the like.

Recently, adaptive hypermedia systems and intellectual multi-agent systems (MAS) have also been used, while designing an agent-based approach based on the use of intellectual agents (programs) [4, 18, 19].

The agent-oriented approach in the construction of intellectual information systems for educational purposes presupposes the creation of information agent-oriented teaching complexes that use virtual worlds of subject domains-complex dynamic models of subject domains that are the closest to the reality of simulated environments and situations [17-19].

The architecture of such complexes is a complex distributed multi-user information system that can be adequately interpreted using multi-level architectural models.

Each level is relatively independent, can be described separately and designed autonomously, and the ways of interaction between the levels are unified.

So, the analysis of existing intellectual information systems for educational purposes showed that the development of systems integrated into the network information and education environment is not given enough attention [2, 3, 12-14, 17]. 
During the research, new approaches to eliminating the shortcomings of existing intellectual information systems and developing expert systems for educational purposes were grounded and proposed.

One of the ways to solve these problems is to create intellectual modules that will be integrated directly into intellectual educational systems, which will ensure: the construction of a sequence of individual teaching courses; intellectual analysis of students' answers; interactive support in solving problems $[2,5,12,21]$.

\section{Justification of the choice of the model of representation and control of knowledge in intellectual teaching systems}

To represent knowledge in intellectual systems (IS), there are different ways $[12,17]$. The presence of various methods is caused primarily by the desire to represent the various types of subject areas with the greatest efficiency. Usually the way of representation in the IS is characterized by the knowledge representation model. The main feature of systems based on knowledge (in particular, intellectual systems) is the availability of a knowledge base in which they are described and the mechanisms for solving problems using this knowledge. Both these components of the teaching systems are in close interconnection. To develop knowledge-based systems, their respective representation is necessary. And this is one of the important problems affecting the characteristics of the teaching system and, ultimately, the quality of knowledge of the students. The choice of a way of representation of knowledge in many respects depends on character and complexity of solved problems. The form of representation of knowledge has a significant impact on the characteristics and properties of teaching systems [12]. The work deals with the representation, storage, processing of large volumes of information, as well as acquiring and using it to gain new knowledge. The process of teaching on the basis of information and communication technologies, we consider as one of the types of information, the basis of which is the semantic dialogue $[5,20]$. For the system under consideration, there arises the need to solve the problem of formalizing the semantic dialogue, which ensures an increase in the effectiveness of its functioning.

In turn, to solve this problem successfully, it is necessary to solve two tasks: formalization of the information process and formalization of semantic information. The formalization of semantic information presupposes the solution of one of the important problems in the theory of information semantic systems, as well as artificial intellect-the representation of knowledge. In addition, for a number of modern subject areas, in particular, computer science is characterized by a constant dynamic, manifested in the emergence of new concepts, structures, concepts, etc. For such subject areas, more flexible ways of presenting knowledge are required. There are various approaches to the problem of the structuring and presentation of knowledge $[5,6,8,9,12,20]$. The method of representation of knowledge offered by us is based on the logical semantic approach, the principles of constructing artificial intellect systems and adaptive information semantic systems. It combines procedural and declarative approach to the representation of knowledge, is based on the theory of semantic networks (SN) and effective algorithms for the logical formation of the output of information about the object on the basis of its belonging to a certain category. To describe semantic networks, there is a semantic language (SL), with which objects $\{\mathrm{Ai}\}$ and relations $\{\mathrm{Ri}\}$ are distinguished [21]. Here $\{\mathrm{Ai}\}$ and $\{\mathrm{Ri}\}$ belong to the set of components $\{\mathrm{SOk}\}$. By components are understood not only objects and relations, but also complex objects and various situations composed of them. Components can also be logical components: truth and falsehood. Components are matched with elements of the same set-vertex. It should be noted that the division of components into objects and relations is conditional. Relationships can be viewed as objects 
related to their relations. Objects can refer to a relationship type. We assume that the role of vertices, which are given by means of tuples, is essential. $<$ so1, ..., sok $>$, where so1, ..., sok $€ \mathrm{SO}$, where $\mathrm{SO}$ is the set of vertex-concepts of the subject domain studied. Tuples are called elementary fragments (EF). In each EF, there are many vertices corresponding to the objects of the domain. At the tops of the teaching SS can be the object of cognition, the personality of the knower and the main components of the teaching process, and the connections between the peaks signify the relationship between them. Among the objects of the semantic network, a hierarchy is established in the relations "is a part" (PartOf), "be a subset," etc. Fig. 1 shows an elementary fragment CC, explaining the concept of hierarchical structures and their purpose.

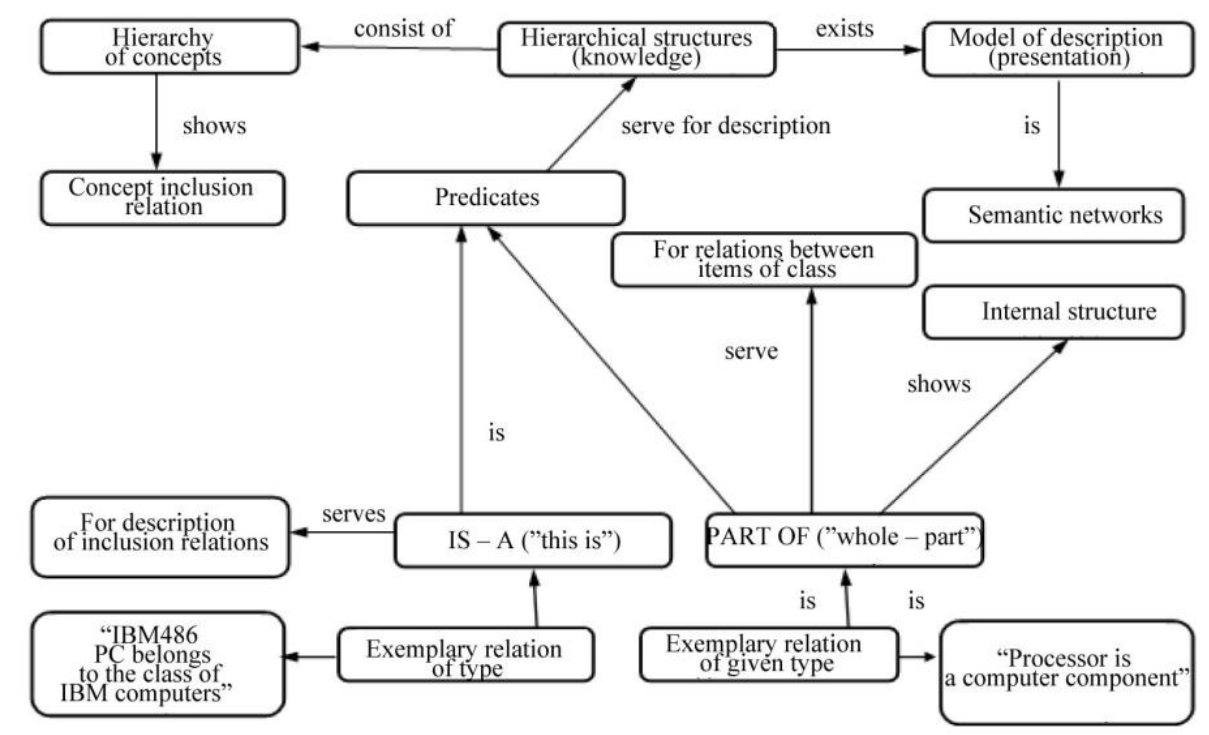

Fig. 1. Semantic model on the topic "Hierarchical structures".

Based on the analysis of existing knowledge engineering approaches [2, 3, 6-8, 23], we chose adaptive semantic models (ASM) as the main way of structuring knowledge in intellectual teaching systems $[12,20]$. The basis of this model is a system of knowledge that has a certain meaning in the form of an integral image of the network, the nodes of which correspond to the concepts and objects of a certain domain, and the arcs to the relations between objects. Adaptive semantic models are also used by us to control students' knowledge and to represent the teaching process itself. The approach we propose is based on the structure of human knowledge, the principles of developing artificial intellect systems and information semantic systems, which is the teaching process. It combines a procedural and declarative approach to the representation of knowledge, is based on the theory of semantic networks and product rules. It should be emphasized that the semantic model, depicting the logical structure of the educational material in accordance with the existing connections between its concepts, simultaneously shows all the basic concepts of the topic under study and the connection between them, which facilitates its perception. As the author's experience in the development of semantic models for some academic disciplines shows, the process of building models promotes the effective acquisition of knowledge. Therefore, students can be taught not only according to the semantic models 
developed by the teacher, but also to assign them tasks for their development, which promotes better teaching of the teaching material.

Figure 2 shows a semantic model for the academic discipline "Mathematical Logic". This academic discipline is abstract. If you can use diagrams, drawings, illustrations to study the components of a personal computer, then the teacher does not have such an opportunity to study abstract disciplines. The lack of visual aids makes it difficult for students to assimilate the content of the subject. The model of educational material on the subject "Algebra of propositions" presented in Fig. 2 presents its basic concepts and shows the cause-effect relations between them.

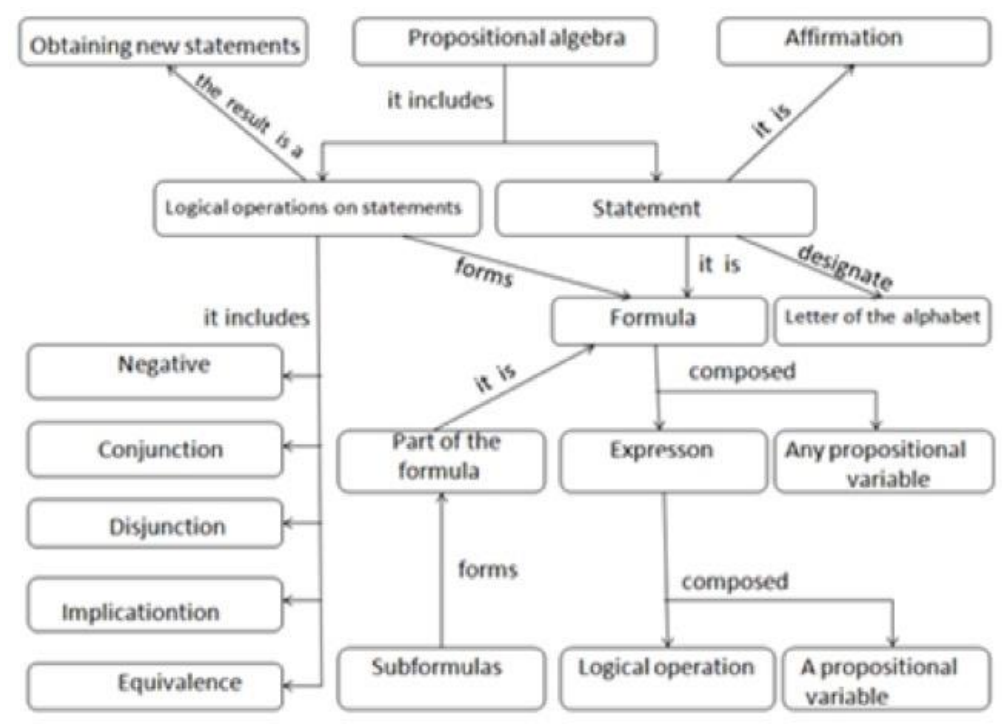

Fig. 2. The Semantic model on the topic "Propositional Algebra".

On the basis of the proposed theoretical approaches, structuring of educational material on some sections of informatics was carried out: programming, computer software, computer modeling, theoretical bases of computer science, mathematical logic, the foundations of artificial intellect and others, on the basis of which a structured and electronic textbook was developed.

As noted above, one of the advantages of semantic networks as a model of representation of knowledge and directly the teaching process itself is the visibility of the description of the subject area, their flexibility and adaptability to the goal and other factors of the teaching process.

However, the visibility with increasing size and complexity of the knowledge base of the domain is lost. In addition, there are significant difficulties in handling various kinds of exceptions. To overcome these problems, the hierarchical description of models is usually used (Fig. 3).

The highest level is taken by classes of concepts, then (the following level) is taken by generalized concepts, and the lowest level is taken by elementary (concrete) concepts. The number of levels of the hierarchical knowledge model of the subject area depends on the degree of detail of the concepts.

This approach to the organization of knowledge in the development of teaching systems shows the interrelationship of the elements of the educational material, allows significantly reducing teaching time, reducing the amount of memory occupied by the knowledge base and data. 
The model in the form of a hierarchical semantic network, being a logical structure of the studied subject area, also shows the sequence of presentation of the educational material.

\section{Level I (classes of concepts)}

Level II (generalized concepts)

Level III (elementary concepts)

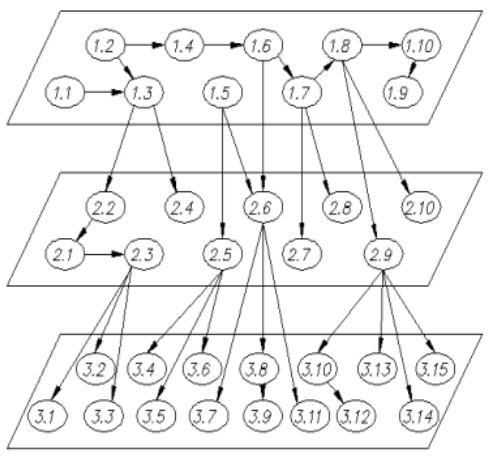

Fig. 3. General multi-level hierarchical model of knowledge representation.

A multi-level hierarchical model of knowledge can be interpreted by an oriented graph. Figure 4 shows the hierarchical model of knowledge in the discipline "Programming".

This model represents various types of concepts (generalized, elementary) of the studied discipline ("Programming"), where the concepts are distributed according to their complexity according to their levels. Thus, at the highest level are the classes of concepts $(\mathrm{CC} 11, \ldots, \mathrm{CCn} 1)$, then the generalized concepts $(\mathrm{GC} 12, \ldots, \mathrm{GCm} 2)$ are placed on the lower level and simpler, elementary concepts (EC31, EC3k).

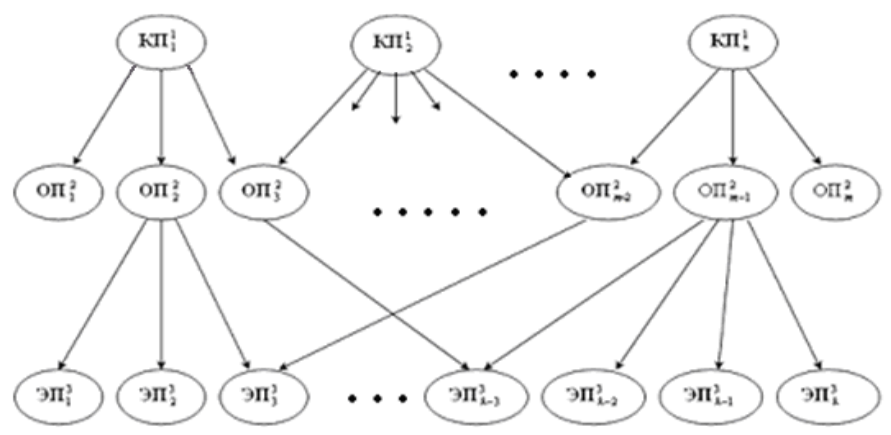

Fig. 4. Representation of the hierarchical model of knowledge oriented graph.

The arrows in Fig. 4 denote such relations between the concepts of the domain as IS-A (as is), PART-OF (is a part), MEMBER-OF (is an element).

$\mathrm{CC}^{1}$ - basic algorithmic structures, $\mathrm{CC}^{1}$ - operators of the Pascal language; $\mathrm{GC}^{1}$ linear algorithms, GC ${ }^{2}$ - cyclic algorithms, $\mathrm{GC}^{3}$ - branching algorithms, $\mathrm{GC}^{m}$ - simple operators, $\mathrm{GC}^{2}-1$ - structural operators; $\mathrm{EC}^{1}$ - cycle with precondition, $\mathrm{EC}^{2}{ }^{3}$ - cycle with postcondition, $\mathrm{EC}^{3}$ - cycle with a specified number of repetitions, $\mathrm{EC}^{k}$ - cycle operator with parameter, $\mathrm{EC}^{3-1}$ - cycle operator with precondition, $\mathrm{EC}^{3-2}$ - cycle operator with a postcondition, $\mathrm{EC}^{k-3}$ - operator of selection. 
To develop multi-level hierarchical adaptive semantic models, the following algorithm was designed:

1. Classification of all concepts of the subject domain under consideration on macroconcepts (class of concepts), metaconcepts (generalized concepts) and microconcepts (elementary concepts).

2. Identification of common properties, features inherent in each level of concepts.

3. Identification of the distinctive features of each level of concepts.

4. Establish links between concepts related to one level.

5. Identification of links between concepts belonging to different levels of the semantic model.

\section{Principles of construction, the architecture of the developed intellectual system of teaching and knowledge control, based on multi-level hierarchical adaptive semantic models}

Based on the proposed theoretical and methodological approaches, a prototype of the intellectual system of teaching and knowledge control [12] was developed, which is used in a number of educational institutions. The software shell of the IOS is implemented in the object - oriented programming environment of Delphi. There are many components for working with databases. Thanks to the component approach to programming, the program can be assembled as a designer, configuring each component to solve a specific task.

Fig. 5 shows the main form of the program, consisting of two pages: Content and User.

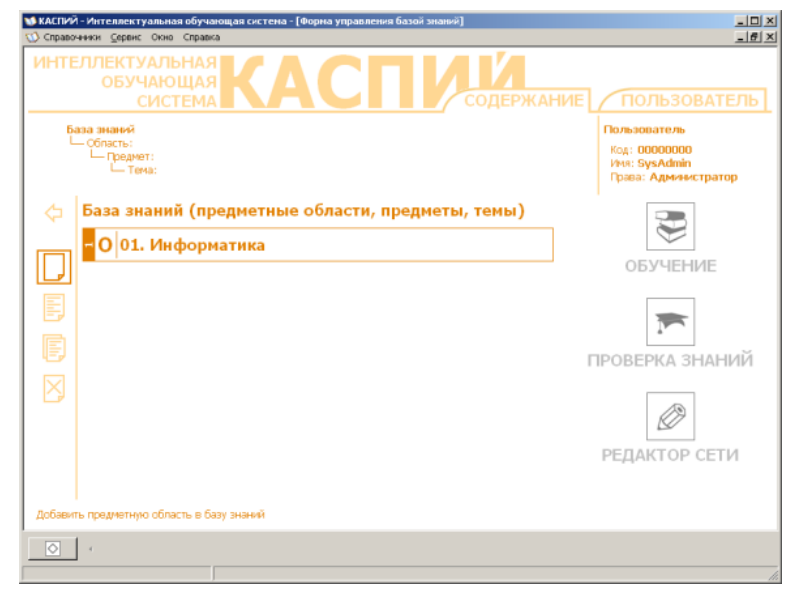

Fig. 5. The main form of the program (page User).

The "Contents" page (Figure 5) displays the navigation history, user settings, the list of knowledge base elements (KB), the list control keys panel and the system model launch keys.

Editing the structure of the knowledge base $(\mathrm{KB})$ is performed on the "Contents" page in the list of elements of the KB. To do this, the form contains keys for managing the list of elements (Figure 5, Table 1). The control keys are available only after the user is registered.

The purpose of each of the keys can also be viewed in the prompt line.

The main component of the page is the list of elements of the database. The subject area, subject, subject and teaching network can act as an element of the base (Fig. 6). These types of elements and their mutual relations form the structure of the BR. The subject area contains subjects that include their themes, and the topics, in turn, contain adaptive semantic models of the logical structure of the educational material to be studied. 
Table 1. Purpose of control keys.

\begin{tabular}{|c|c|c|}
\hline Key & Name & Purpose \\
\hline & Return & Return to preceding level \\
\hline & Add & Addition of new element \\
\hline Edit & $\begin{array}{c}\text { Editing parameters of selected } \\
\text { element }\end{array}$ \\
\hline Copy & $\begin{array}{c}\text { Create a new element with } \\
\text { copying parameters of selected } \\
\text { element }\end{array}$ \\
\hline
\end{tabular}

The elements of the KB are displayed in the form of a list table. Each item in the list includes the following information:

1) number of the topic under study in the list (indicated by the vertical font);

2) subject area, subject, subject and semantic model, corresponding to the topic under study.

On the right side of the "Contents" page there are keys for starting the system modules (Fig. 5). The color of the keys changes depending on the type of the selected list item. In the "User" page (Figure 6) there is a user registration panel in the system, a list of user's work history, and user parameters. In addition, the knowledge base of the system under consideration can be supplemented with subject areas, specific teaching courses and new educational topics to be studied.

During the teaching, the intellectual system is used in the modes: "Edit," "Teaching," "Knowledge control." The module "Network Editor" is intended for building teaching networks, saving them in a database, creating test tasks for testing knowledge.

At the stage of the "Edit" mode, the problem is that the oriented knowledge bases of educational disciplines are formed on the basis of adaptive semantic models. The teaching material in the IOS is studied using the "Teaching" module, and the "Knowledge Control" module has been developed to test students' knowledge. Figure 6 shows the program window when editing a test task.

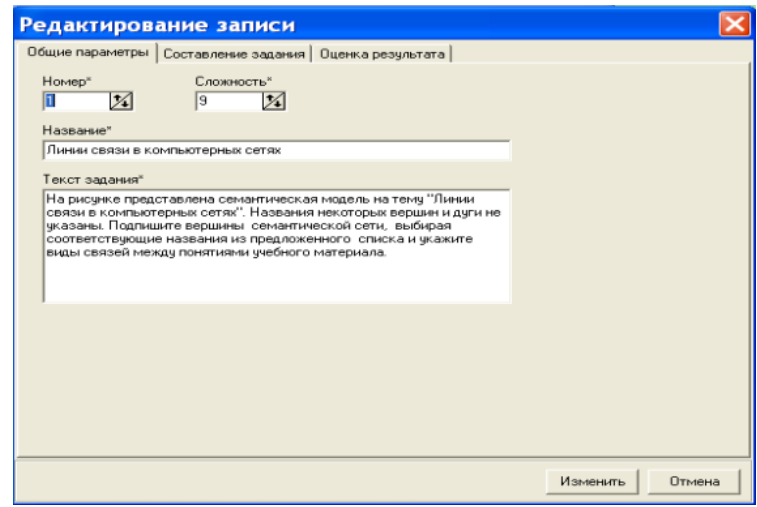

Fig. 6. The window for editing the control task.

To start the "Knowledge Check" module, select the teaching network in the list of PO items and press the key. The form of the "Knowledge Check" module is shown in Fig. 7. The form consists of a toolbar, a timer, a task text panel and a network editing area. 


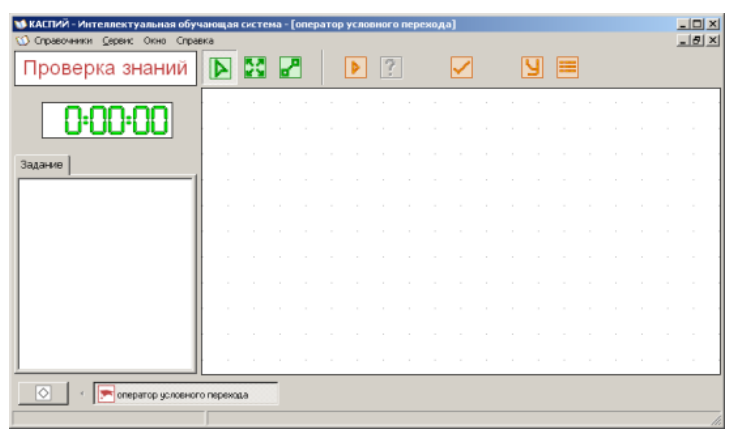

Fig. 7. Kind of the form of the module "Knowledge control."

When the testing process starts, the Knowledge Control module performs initial initialization of the network according to the task. Depending on the type of task, network nodes can be randomly placed in the editing area or arranged without changing the location. Nodes of nodes may also be absent. In this case, the user will need to enter a name for all nodes. Also it is necessary to enter the names of the links, if this is provided by the task (Fig. 8).

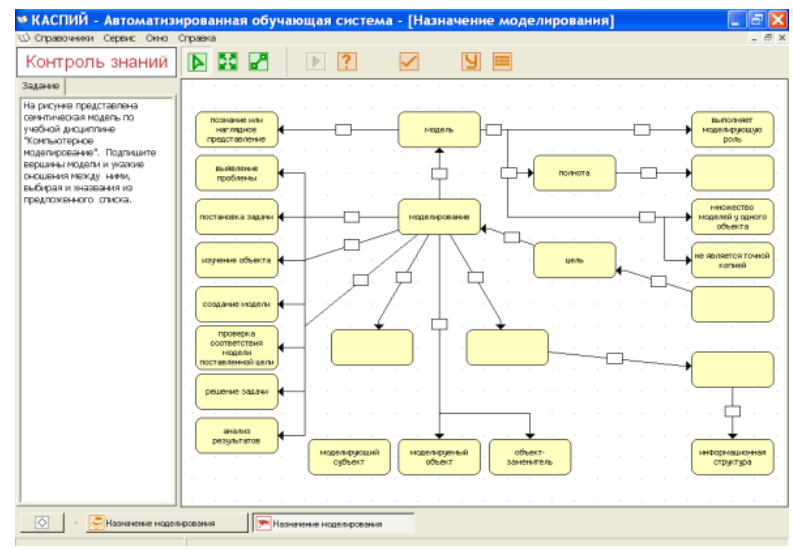

Fig. 8. View the form of the Knowledge Control module after starting the testing process.

The timer panel counts the test time. In the area of editing the form of the "Knowledge Control" module, the user collects the network. In the example task, shown in Fig. 9 it is necessary to sign the vertices of the semantic model and the relations between them, choosing them from the proposed list. If necessary, the nodes are arranged in object management mode. The interconnection between nodes is performed in the add connection mode. After the network is assembled, i.e. all links, all names of nodes and links are placed, you can run a check (check key). The Knowledge Check module checks the user's network by comparing it with the one in the database.

After checking the incorrectly assembled parts of the network will be marked in red. Also, immediately after the verification, a detailed network verification report is issued, indicating the student's grade, mistakes, a topic he has not learned well.

The Knowledge Control module fixes the following types of errors:

1) The error of the arrangement of nodes means that the node is or is not properly connected to other nodes;

2) The error of the node name means that the wrong name has been selected for the node; 
3) The node level error means that the selected name for the node does not match its level;

4) The communication error means that the connection is incorrect or that there is no connection where it should be. At the same time, for both linked nodes, the node allocation error is set;

5) The communication type error means that the connection name is not selected correctly.

When monitoring knowledge, you can also use the query network $[5,12]$.

It should be noted that as the content of the teaching disciplines replenishes with new concepts, the methodology of using IES in the educational process provides for the editing of adaptive semantic models of educational material. There is both an autonomous and a network version of the use of the developed system of teaching and knowledge control and it is invariant with respect to specific academic disciplines.

\section{Conclusion}

The above information, considerations and recommendations allowed systematizing and summarizing the main methodological provisions for the presentation and control of knowledge in educational systems using adaptive semantic models.

1. To represent and control knowledge in educational systems, it is expedient to use adaptive semantic models as the main models providing these tasks, taking into account their possibilities for more adequate description of the interaction of various concepts and sections of educational disciplines and their adaptation to a rapid change in the content of these disciplines and the level of knowledge students.

2. Adaptive semantic models provide a deep structuring of the concepts and phenomena studied, the subject area can be visualized in the form of complex hierarchical models that can identify the knowledge of students and their abilities, make full use of the achievements of modern artificial intellect systems.

3. The main stages of the structuring of knowledge in adaptive semantic models in most cases can be considered:

- Definition of input and output data;

- Compiling a vocabulary of terms;

- Identification of objects and concepts;

- Identification of links between concepts;

- Identification of meta-concepts and the specification of concepts;

- Building knowledge pyramids;

- Definition of relations between concepts;

- Definition of decision making strategy.

4. When constructing knowledge pyramids, visual materials should be used: drawings, diagrams, diagrams, graphics, etc.; the levels of the knowledge pyramid often arise in the minds of students in the form of visual images.

5. One of the main methods (apparatus) of knowledge structuring is psychosemantics, which allows you to explore the structures of consciousness through the reconstruction of an individual knowledge system, revealing categorical structures of experts' consciousness.

6 . In connection with the fact that the property of visibility of ACM when using multidimensional knowledge bases and the complication of connections between its objects, it is useless to use multi-level hierarchical adaptive semantic models in which concepts and objects of the domain are located on several levels.

7. The development of educational ACMs for specific academic disciplines is recommended in the following order:

- classification of concepts in the subject area; 
- highlighting common properties and attributes inherent in each level of teaching concepts;

- $\quad$ highlighting the distinctive features of each level of concepts;

- establishing links between concepts related to the same level;

- highlight inter-level and intersubject communications.

8. Controlling the knowledge of students on the basis of AFM should presuppose a semantic processing of their answers and a comparison of the knowledge of the students with the data of the educational ACM, while the network for requesting educational information can be used and the activation of student teaching activities and the objectivity of monitoring their knowledge should be ensured.

10. The procedure for the synthesis of tests to monitor the knowledge of students should provide the maximum possible information about the subject area in the responses of students with the minimum possible number of tests.

It should be noted that the developed methodology of knowledge control provides: an expedient sequence of presentation of control tasks to the learner; the possibility of using an activity approach to the process of testing knowledge and using more complex and complicated problems in the subject area, including in problematic situations; while the network for requesting educational information ensures that the content of the control tasks corresponds to the study material studied. The above information, considerations and recommendations allowed systematizing and summarizing the main methodological provisions for the presentation and control of knowledge in educational systems using adaptive semantic models.

1. To represent and control knowledge in educational systems, it is expedient to use adaptive semantic models as the main models providing these tasks, taking into account their possibilities for more adequate description of the interaction of various concepts and sections of educational disciplines and their adaptation to a rapid change in the content of these disciplines and the level of knowledge of students.

2. Adaptive semantic models provide a deep structuring of the concepts and phenomena studied, the subject area can be visualized in the form of complex hierarchical models that can identify the knowledge of students and their abilities, make full use of the achievements of modern artificial intellect systems.

3. The main stages of the structuring of knowledge in adaptive semantic models in most cases can be considered:

- Definition of input and output data;

- Compiling a vocabulary of terms;

- Identification of objects and concepts;

- Identification of links between concepts;

- Identification of meta-concepts and the specification of concepts;

- Building knowledge pyramids;

- Definition of relations between concepts;

- Definition of decision making strategy.

4. When constructing knowledge pyramids, visual materials should be used: drawings, diagrams, diagrams, graphics, etc.; the levels of the knowledge pyramid often arise in the minds of students in the form of visual images.

5. One of the main methods (apparatus) of knowledge structuring is psychosemantics, which allows you to explore the structures of consciousness through the reconstruction of an individual knowledge system, revealing categorical structures of experts' consciousness.

6. In connection with the fact that the property of visibility of ACM when using multidimensional knowledge bases and the complication of connections between its objects, it is useless to use multi-level hierarchical adaptive semantic models in which concepts and objects of the domain are located on several levels. 
7. The development of educational ACMs for specific academic disciplines is recommended in the following order:

- classification of concepts in the subject area;

- highlighting common properties and attributes inherent in each level of teaching concepts;

- highlighting the distinctive features of each level of concepts;

- establishing links between concepts related to the same level;

- highlight inter-level and intersubject communications.

8. Controlling the knowledge of students on the basis of AFM should presuppose a semantic processing of their answers and a comparison of the knowledge of the students with the data of the educational ACM, while the network for requesting educational information can be used and the activation of student teaching activities and the objectivity of monitoring their knowledge should be ensured.

10. The procedure for the synthesis of tests to monitor the knowledge of students should provide the maximum possible information about the subject area in the responses of students with the minimum possible number of tests.

It should be noted that the developed methodology of knowledge control provides: an expedient sequence of presentation of control tasks to the learner; the possibility of using an activity approach to the process of testing knowledge and using more complex and complicated problems in the subject area, including in problematic situations; while the network for requesting educational information ensures that the content of the control tasks corresponds to the study material studied.

\section{References}

1. J. A. Vagramenko, Managing Education: Theory and Practice, 4, 24 (2016)

2. J. A. Vagramenko, Informatization of Education and Science, 4, 32 (2016)

3. S. G. Danyluk, Scientific Notes of IIO RAO, 50 (2013)

4. G. V. Rybina, Information-Measuring and Control System, 10 (2011)

5. T. S. Shikhnabieva, Pedagogical Informatics, 4 (2014)

6. L. Jain, X Wu, Advanced information and knowledge processing (Springer, London, 2009)

7. E. Wenger, Artificial intelligence and tutoring systems: computational approaches to the communication of knowledge (Morgan Kaufmann, Los Altos 1987)

8. V. L. Uskov, A. V. Uskov, Advanced Technology for Learning, 5, 1 (2007)

9. V. L. Uskov, A. V. Uskov, Advanced Technology for Learning, 4, 3 (2007)

10. M. Reichert, R. Oberhauser, G. Grambow, Advances in intelligent process-aware information systems (Springer, 2017)

11. Ya. A. Vagramenko, G. Yu. Yalamov, Pedagogical Informatics, 3 (2015)

12. T. Shikhnabieva, S. Beshenkov, Smart education and e-learning (Springer, 2016)

13. M. Rod, Int. conf. adv. res. comput. educ. (Tokyo, 1990)

14. E. Szczerbicki, M. Graña, J. Posada, C. Toro, Current Research Advances and Implementations in Smart Knowledge-Based Systems - Part I: Cybernetics and Systems, 44, 2-3 (2013)

15. M. Graña, C. Toro, J. Posada, R. J. Howlett, L. C. Jain, Frontiers in Artificial Intelligence and Applications, 243 (2012) 
16. EDUCASE Center for Applied Research, The ECAR study of Undergraduate Students and Information Technology, 7 (2006)

17. V. L. Uskov, A. V. Uskov, Advanced Technology for Learning, 4, 3 (2007)

18. A. N. Shvetsov, Information Technologies in Design and Production, 1 (2010)

19. A. N. Shvetsov, E. V. Sibirtsev, I. A. Andrianov, The XII all-Russian meeting on problems of management VSPU-2014 (http://vspu2014.ipu.ru/proceedings /vspu2014.zip)

20. T. Sh. Shikhnabiyeva, Management of Education: Theory and Practice, 3, 27 (2017)

21. T. Sh. Shikhnabieva, Information Environment of Education and Science, 17 (2013)

22. I. Kuznetsov, Semantic representations (Science, Moscow, 1986)

23. T. A. Gavrilova, V. F. Khoroshevsky, Knowledge base of intellectual systems (Peter, St. Petersburg, 2000) 